\title{
Model of Persistent Breaking of Discrete Symmetry
}

\author{
Noam Chai $\odot,{ }^{1}$ Anatoly Dymarsky, ${ }^{2,3}$ and Michael Smolkin $\circledast^{1}$ \\ ${ }^{1}$ The Racah Institute of Physics, The Hebrew University of Jerusalem, Jerusalem 91904, Israel \\ ${ }^{2}$ Department of Physics and Astronomy, University of Kentucky, Lexington, Kentucky 40506, USA \\ ${ }^{3}$ Skolkovo Institute of Science and Technology, Skolkovo Innovation Center, Moscow 143026, Russia
}

(Received 30 July 2021; accepted 20 December 2021; published 3 January 2022)

\begin{abstract}
We show there exist UV-complete field-theoretic models in general dimension, including $2+1$, with the spontaneous breaking of a global symmetry, which persists to the arbitrarily high temperatures. Our example is a conformal vector model with the $O(N) \times \mathbb{Z}_{2}$ symmetry at zero temperature. Using conformal perturbation theory we establish $\mathbb{Z}_{2}$ symmetry is broken at finite temperature for $N>17$. Similar to recent constructions of [N. Chai et al., Phys. Rev. D 102, 065014 (2020)., N. Chai et al., Phys. Rev. Lett. 125, 131603 (2020).], in the infinite $N$ limit our model has a nontrivial conformal manifold, a moduli space of vacua, which gets deformed at finite temperature. Furthermore, in this regime the model admits a persistent breaking of $O(N)$ in $2+1$ dimensions, therefore providing another example where the ColemanHohenberg-Mermin-Wagner theorem can be bypassed.
\end{abstract}

DOI: 10.1103/PhysRevLett.128.011601

Introduction.-The phenomenon of spontaneous symmetry breaking is ubiquitous: many real systems as well as field theoretic models exhibit spontaneous breaking of both discrete and continuous symmetries at zero or sufficiently small temperature. The conventional picture suggests the full symmetry is restored for sufficiently high temperatures. There are also situations when the low-temperature phase is symmetric but symmetry is broken as the temperature increases. In this case too one normally expects the symmetry to be eventually restored for even higher temperatures, and there are seemingly many theoretical results supporting such a conclusion. Yet as we discuss below they rely on the stringent assumptions that can be evaded. Which raises the question-is spontaneous breaking that persists to arbitrarily high temperatures possible? In this Letter we answer this question by constructing UV-complete field-theoretical models in diverse dimensions which exhibit persistent breaking of both discrete and continuous symmetries.

For lattice systems it is well appreciated the symmetry is restored for temperates large in comparison with the lattice spacing [1]. This suggests in effective field theory spontaneous breaking is possible up to UV scale, as illustrated by the UV-incomplete example of Ref. [2]. Yet the latticebased arguments are not applicable to UV-complete field theoretic models, which we focus on. There is also the

Published by the American Physical Society under the terms of the Creative Commons Attribution 4.0 International license. Further distribution of this work must maintain attribution to the author(s) and the published article's title, journal citation, and DOI. Funded by SCOAP ${ }^{3}$. famous Coleman-Hohenberg-Mermin-Wagner theorem $[3,4]$ and its generalizations, which rule out the possibility of the longer range order at $T \neq 0$ in a wide class of twodimensional $d=2+1$ systems. This seemingly prohibits spontaneous breaking of a continuous symmetry, but here too there are many assumptions and exceptions, starting from the example of Ref. [5]. Importantly to what follows, the original work [4] already notes the argument may break down if the lattice model exhibits long-range interactions. Thus, there is a phase transition in a Heisenberg magnet with suitably adjusted long-range interactions, see, e.g., Ref. [6] for a recent review on the Coleman-HohenbergMermin-Wagner theorem and its limitations. This suggests nonlocal field theories, which may result from such lattice models in the continuous limit may be immune to various no-go results.

To better illustrate this idea we briefly mention Coleman's no-go theorem [7], which in $d=1+1$ excludes spontaneous symmetry breaking because the corresponding Goldstone bosons, being massless, would have infrared divergences. This argument works for the short-range interactions, whereas as above introduction of the longrange forces allows phase-transitions in the one-dimensional systems [8-12]. The no-go results in various dimensions are related and can be evaded simultaneously: Our model exhibits persistent breaking for $1<d<3+1$.

The discussion above mostly applies to continuous symmetries. For discrete symmetries even less is known. As for continuous symmetries, there is no universal theoretical argument requiring discrete symmetry to be restored at high temperatures. At the same time to the best of our knowledge there were no examples of persistent symmetry breaking in $2+1$ dimensions. In higher 
dimensions there are CFTs in $d=4-\epsilon$ [13-15] and $d=4$ $[16,17]$ which have some of their internal symmetries broken at arbitrary finite temperature. Yet these models are not free of criticism. In the former case $\epsilon$ cannot be taken to one and therefore theories in question are not necessarily unitary [18]. And the latter case of [16] is inconclusive because of the possible impact of $1 / N$ corrections. We also refer the reader to Ref. [19], where asymptotically safe theories [20] in $d=3+1$ were considered in the context of persistent symmetry breaking. Another idea explored in the literature is placing the theory in a curved spacetime. Thus, the $O(N)$ model in AdS evades the Coleman-Hohenberg-Mermin-Wagner theorem [21] but at high temperatures the symmetry is restored. There are other AdS/CFT candidates of persistent order [22-28] which are perturbatively stable, but the symmetric phase has smaller free energy. (See Ref. [29] where the holographic conformal order is studied on $S^{3}$.) Other nonunitary models of persistent breaking in the presence of chemical potential include [30-35]. We should also mention the renowned Berezinskii-Kosterlitz-Thouless transition [36-38] as an example evading the ColemanHohenberg-Mermin-Wagner theorem yet not leading to persistent symmetry breaking.

The main goal of this Letter is to construct an example which would be free of aforementioned deficiencies. Our model is a conformal, and hence UV-complete vector theory which breaks discrete symmetry at finite temperature $T$. Because of scale invariance this breaking persists to arbitrary high $T$. The result holds true in $2+1$ and extends to other $1<d<3+1$ where the model is manifestly stable (Stability suggests the model is unitary; it would be interesting to confirm this by calculating anomalous dimensions at the IR fixed point. We also expect the fixed point to be a CFT, but since our model is nonlocal strictly speaking this has to be verified along the lines [39].) Generalizations of our model exhibit persistent breaking of continuous symmetries in $d=2+1$ and beyond [40]. Our construction bypasses the Coleman-Hohenberg-MerminWagner theorem due to its nonlocal nature. It can be viewed as two weakly interacting copies of the long-range Ising (LRI) model $[8,41,42]$ (For original and recent studies of the LRI model see Refs. [39,43-51].)

The model.-To begin with, we introduce our model. Consider the following Gaussian action in $1 \leq d<4$ dimensions,

$$
\begin{aligned}
S_{0}= & \mathcal{N}_{\phi} \int d^{d} x_{1} \int d^{d} x_{2} \frac{\vec{\phi}\left(x_{1}\right) \cdot \vec{\phi}\left(x_{2}\right)}{\left|x_{1}-x_{2}\right|^{2\left(d-\Delta_{\phi}\right)}} \\
& +\mathcal{N}_{\sigma} \int d^{d} x_{1} \int d^{d} x_{2} \frac{\sigma\left(x_{1}\right) \sigma\left(x_{2}\right)}{\left|x_{1}-x_{2}\right|^{2\left(d-\Delta_{\sigma}\right)}} .
\end{aligned}
$$

The fundamental fields include scalars $\vec{\phi}$ and $\sigma$ transforming in vector and singlet representations of $O(N)$. The model also admits a $\mathbb{Z}_{2}$ symmetry that flips the sign of $\sigma$. The coefficients $\mathcal{N}_{\phi}$ and $\mathcal{N}_{\sigma}$ are fixed so that the two-point functions of $\vec{\phi}$ and $\sigma$ are canonically normalized. For brevity we suppress vector indices in what follows. The scaling dimensions of the generalized free fields are chosen to be

$$
\Delta_{\phi}=\frac{d-\epsilon_{1}}{4}, \quad \Delta_{\sigma}=\frac{d-\epsilon_{3}}{4},
$$

with $\epsilon_{1,3} \ll 1$, so that the following quartic operators become weakly relevant

$$
\mathcal{O}_{1}=\left(\phi^{2}\right)^{2}, \quad \mathcal{O}_{2}=\phi^{2} \sigma^{2}, \quad \mathcal{O}_{3}=\sigma^{4},
$$

with scaling dimensions $\Delta_{1}=4 \Delta_{\phi}, \Delta_{2}=2\left(\Delta_{\phi}+\Delta_{\sigma}\right)$, and $\Delta_{3}=4 \Delta_{\sigma}$. This model is conformal, we list OPE coefficients $C_{i j}^{k}$ and other technical details in the Appendix.

Next, we consider the following deformation

$$
S=S_{0}+\sum_{i=1}^{3} \frac{g_{i} \mu^{\epsilon_{i}}}{N} \int d^{d} x \mathcal{O}_{i}(x),
$$

where $\epsilon_{i} \ll 1$. [Note that $\epsilon_{2}=\left(\epsilon_{1}+\epsilon_{3}\right) / 2$.] It induces an $\mathrm{RG}$ flow of the form

$$
\mu \frac{d g_{i}}{d \mu}=-\epsilon_{i} g_{i}+\frac{\pi^{d / 2}}{N \Gamma\left(\frac{d}{2}\right)} \sum_{j, k} C_{j k}^{i} g_{j} g_{k}+\ldots
$$

As we argue below, for $N>10$ there is a fixed point with $g_{2}<0$. Moreover, the IR CFTs with negative $g_{2}$ are stable. They define a class of theories with the persistent symmetry breaking.

To understand the unbroken symmetries of the IR critical point at finite temperature we consider the effective potential $V_{\text {eff }}$ for the zero mode. To leading order in $\epsilon_{i}$, thermal fluctuations simply induce quadratic terms in addition to the quartic potential (4),

$$
\begin{aligned}
V_{\text {eff }}(\phi, \sigma ; \beta)= & \mathcal{M}_{\phi}(\beta) \phi^{2}+\mathcal{M}_{\sigma}(\beta) \sigma^{2} \\
& +\frac{g_{1} \mu^{\epsilon_{1}}}{N} \mathcal{O}_{1}+\frac{g_{2} \mu^{\epsilon_{2}}}{N} \mathcal{O}_{2}+\frac{g_{3} \mu^{\epsilon_{3}}}{N} \mathcal{O}_{3}+\mathcal{O}\left(\epsilon_{i}^{2}\right),
\end{aligned}
$$

where

$$
\begin{aligned}
& \mathcal{M}_{\phi}(\beta)=2 \frac{g_{1} \mu^{\epsilon_{1}}}{N}\left(1+\frac{2}{N}\right)\left\langle\phi^{2}\right\rangle_{\beta}+\frac{g_{2} \mu^{\epsilon_{2}}}{N}\left\langle\sigma^{2}\right\rangle_{\beta}, \\
& \mathcal{M}_{\sigma}(\beta)=\frac{g_{2} \mu^{\epsilon_{2}}}{N}\left\langle\phi^{2}\right\rangle_{\beta}+6 \frac{g_{3} \mu^{\epsilon_{3}}}{N}\left\langle\sigma^{2}\right\rangle_{\beta} .
\end{aligned}
$$

As shown in the Appendix, thermal expectation values of the generalized free fields are given by 


$$
\left\langle\phi^{2}\right\rangle_{\beta}=N \frac{2 \zeta\left(2 \Delta_{\phi}\right)}{\beta^{2 \Delta_{\phi}}}, \quad\left\langle\sigma^{2}\right\rangle_{\beta}=\frac{2 \zeta\left(2 \Delta_{\sigma}\right)}{\beta^{2 \Delta_{\sigma}}}
$$

which follow straightforwardly from the thermal Green's function. Obviously, full $O(N) \times \mathbb{Z}_{2}$ symmetry is preserved by the minimum of the potential at zero temperature. However, the finite temperature effects might break it if $\mathcal{M}_{\phi}<0$ or $\mathcal{M}_{\sigma}<0$. If this happens, the higher order perturbative corrections cannot restore the symmetry, because the higher loop contributions to $\mathcal{M}_{\phi}, \mathcal{M}_{\sigma}$ are suppressed by higher powers of $\epsilon_{i}$, whereas the terms with higher powers of fundamental fields are subdominant in the vicinity of the origin. To establish symmetry breaking at finite temperature, it is therefore sufficient to show that the model admits a fixed point where one of the $\mathcal{M}$ 's becomes negative.

To ensure stability of the model it is necessary to satisfy $g_{1}, g_{3} \geq 0$, while $g_{2}$ could be negative provided $4 g_{1} g_{3} \geq g_{2}^{2}$. If all $g_{i}$ are positive, $\mathcal{M}_{\phi}, \mathcal{M}_{\sigma}$ are positive as well, the potential is minimized at the origin $\phi=\sigma=0$ and the symmetry is restored. The only scenario of symmetry breaking is therefore when $g_{2}<0$. The RG flow (5) terminates at a weakly interacting IR fixed point in the vicinity of the original Gaussian theory. At the critical point at leading order in $\epsilon_{i}$ the couplings satisfy

$$
\begin{aligned}
& \epsilon_{1} g_{1}=\frac{\pi^{d / 2}}{N \Gamma\left(\frac{d}{2}\right)}\left(C_{11}^{1} g_{1}^{2}+C_{22}^{1} g_{2}^{2}\right), \\
& \epsilon_{2} g_{2}=\frac{\pi^{d / 2}}{N \Gamma\left(\frac{d}{2}\right)}\left(2 C_{12}^{2} g_{1} g_{2}+C_{22}^{2} g_{2}^{2}+2 C_{23}^{2} g_{2} g_{3}\right), \\
& \epsilon_{3} g_{3}=\frac{\pi^{d / 2}}{N \Gamma\left(\frac{d}{2}\right)}\left(C_{22}^{3} g_{2}^{2}+C_{33}^{3} g_{3}^{2}\right) .
\end{aligned}
$$

There is always a trivial fixed point with $g_{1}=(\Gamma(d / 2) /$ $\left.8 \pi^{d / 2}\right)(N / N+8) \epsilon_{1}, g_{2}=0$ and $g_{3}=\left(N \Gamma(d / 2) / 72 \pi^{d / 2}\right) \epsilon_{3}$. It represents two decoupled theories: the so-called long range Ising model [39] and its $O(N)$ generalization. This fixed point was recently studied in, e.g., Ref. [51].

To simplify the analysis and illustrate the main idea in what follows we consider only a particular case of equal $\epsilon_{i}=\epsilon$. The case of nonequal $\epsilon_{i}$ is similar and also admits persistent symmetry breaking. It is also convenient to rescale the couplings $g_{i}=\tilde{g}_{i}\left(\Gamma(d / 2) / \pi^{d / 2}\right) \epsilon_{i}$. Before proceeding with the case of finite $N$ we take the infinite $N$ limit. In this case the equations (9) drastically simplify, yielding the conformal manifold-a one-parameter family of fixed points

$$
\tilde{g}_{1}=\frac{1}{8}, \quad \tilde{g}_{3}=2 \tilde{g}_{2}^{2},
$$

depicted in Fig. 1. For the negative branch $\tilde{g}_{2}=-2 \sqrt{\tilde{g}_{1} \tilde{g}_{3}}$ the effective potential degenerates into

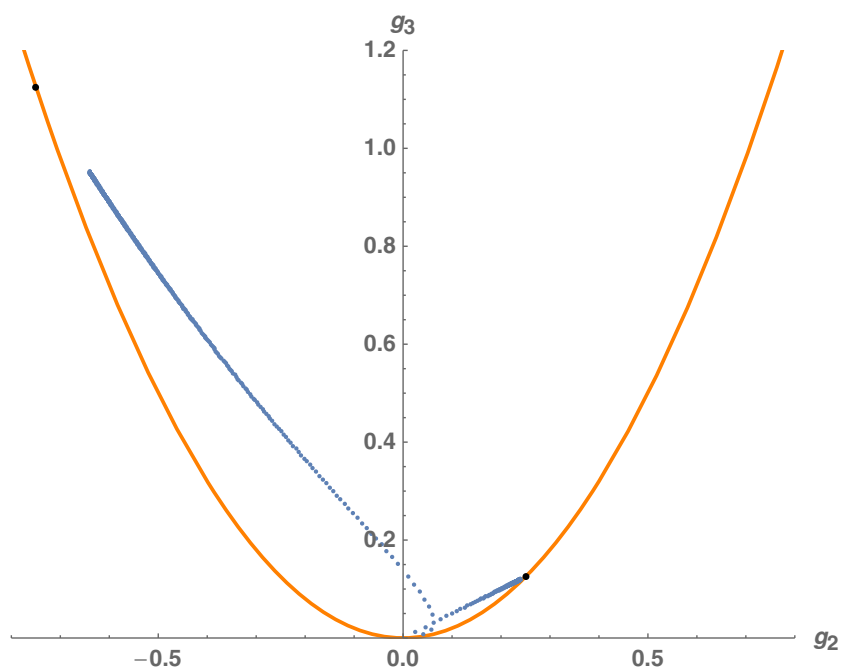

FIG. 1. Fixed points in the $g_{2}-g_{3}$ plane. Orange line shows a continuous family of solutions (10) emerging in the infinite $N$ approximation. Black dots are the asymptotic locations of fixed points (14) in the limit of large $N$. Blue points are the solutions of Eq. (9) for $N \leq 200$.

$$
\begin{gathered}
V_{\mathrm{eff}}(\phi, \sigma ; \beta)=\frac{\mu^{\epsilon}}{N}\left(2 \sqrt{g_{1}}\left\langle\phi^{2}\right\rangle_{\beta} x+x^{2}\right), \\
x=\sqrt{g_{1}} \phi^{2}-\sqrt{g_{3}} \sigma^{2} .
\end{gathered}
$$

The minimum is reached at

$$
\sigma^{2}=\sqrt{\frac{g_{1}}{g_{3}}}\left(\phi^{2}+\left\langle\phi^{2}\right\rangle_{\beta}\right) .
$$

This is a one-dimensional family of vacua with the nonzero expectation value of $\sigma$, signaling spontaneous symmetry breaking. For positive $g_{2}$ and $\left\langle\phi^{2}\right\rangle_{\beta}$ only $\sigma=0$ is admitted.

From the discussion above it is clear negative $g_{2}$ is necessary for symmetry to be broken. Hence the crucial question is if the fixed point(s) with $g_{2}<0$ survive in the finite $N$ regime. Before proceeding with an arbitrary $N$ we employ $1 / N$ expansion to find, in addition to Eq. (10), the consistency condition

$$
4 \tilde{g}_{2}^{2}+2 \tilde{g}_{2}-\frac{3}{4}=0
$$

Hence at large but finite $N$ the continuous family (10) collapses into two solutions, one with positive and one with negative $g_{2}$. Upon taking finite $N$ corrections into account, degeneracy $4 g_{1} g_{3}=g_{2}^{2}$ is lifted. Minimization of the potential (6) yields

$$
\left(\begin{array}{l}
\phi^{2} \\
\sigma^{2}
\end{array}\right)=\frac{-N \mu^{-\epsilon}}{4 g_{1} g_{3}-g_{2}^{2}}\left(\begin{array}{cc}
2 g_{3} & -g_{2} \\
-g_{2} & 2 g_{1}
\end{array}\right)\left(\begin{array}{l}
\mathcal{M}_{\phi} \\
\mathcal{M}_{\sigma}
\end{array}\right),
$$

provided resulting $\phi^{2}$ and $\sigma^{2}$ both are positive. Yet this is never the case for any solutions of Eq. (9). The true 
minimum of the potential is therefore achieved either at $\phi^{2}=0$ or $\sigma^{2}=0$. For all solutions of Eq. (9) $\mathcal{M}_{\phi}>0$ but $\mathcal{M}_{\sigma}$ become negative for the branch with negative $g_{2}$ and $N>17$. In these cases the minimum is achieved at

$$
\left(\begin{array}{c}
\phi^{2} \\
\sigma^{2}
\end{array}\right)=\frac{-N \mu^{-\epsilon}}{2 g_{3}}\left(\begin{array}{c}
0 \\
\mathcal{M}_{\sigma}
\end{array}\right)
$$

At leading order in $\epsilon$ this value is correct at all temperatures, as follows from the dimensional analysis. Clearly, $\sigma^{2}$ is strictly positive, indicating symmetry breaking.

Conclusions.-To summarize, the model (1) with the choice $\epsilon_{i}=\epsilon \ll 1$ for finite $N$ has two different IR fixed points, and for $N>10$ one of them has negative $g_{2}$. The fixed points with $g_{2}<0$ and $N>17$ exhibit symmetry breaking at arbitrary nonzero temperature

$$
O(N) \times \mathbb{Z}_{2} \rightarrow O(N) .
$$

The behavior for nonequal small $\epsilon_{i}$ is qualitatively similar.

The model (1) admits a straightforward generalization to two Gaussian free fields in the vector representations of $O\left(N_{1}\right)$ and $O\left(N_{2}\right)$. The example in this note corresponds to the $N_{2}=1$ case. Deforming this theory by weakly relevant quartic operators and following the same steps as above, one finds IR fixed points which exhibit spontaneous breaking of the global continuous symmetry at finite temperature. In $2+1$ dimensions it is therefore an example of persistent breaking of a continuous global symmetry, which bypasses the Coleman-Hohenberg-Mermin-Wagner theorem $[3,4,7]$ by virtue of being a CFT with long-range interactions. We will discuss this case in detail in Ref. [40]. Our nonlocal $O(N) \times \mathbb{Z}_{2}$ model is similar to that one studied in Refs. $[13,14]$. It would be also interesting to explore if they are related in the large $N$ limit.

Our findings clearly show persistent breaking is possible in the UV complete yet nonlocal models. This raises the question if nonlocality is truly necessary, i.e., if there could be UV-complete unitary local field theoretic models in $d=$ $2+1$ exhibiting persistent breaking of discrete symmetries. It is an important open question to construct such an example or rule out this possibility.

We thank Alex Avdoshkin, Dean Carmi, Soumyadeep Chaudhuri, Changha Choi, Joshua Feinberg, Mikhail Goykhman, Eliezer Rabinovici, Ritam Sinha, and especially Zohar Komargodski for helpful discussions and correspondence. This work is partially supported by the Binational Science Foundation (Grant No. 2016186). A. D. is grateful to Weizmann Institute of Science for hospitality and acknowledges sabbatical support of the Schwartz/ Reisman Institute for Theoretical Physics. N.C. and M.S. are grateful to the Israeli Science Foundation Center of Excellence (Grant No. 2289/18) and the Quantum Universe I-CORE program of the Israel
Planning and Budgeting Committee (Grant No. 1937/12) for continuous support of our research. N. C. is grateful for the support from the Yuri Milner scholarship.

Appendix: Supplemental Material.-The action defines a conformal model with the canonically normalized twopoint function of fundamental fields $\vec{\phi}$ and $\sigma$,

$$
\left\langle\phi_{a} \phi_{b}\right\rangle=\frac{\delta_{a b}}{\left|x_{12}\right|^{2 \Delta_{\phi}}}, \quad\langle\sigma \sigma\rangle=\frac{1}{\left|x_{12}\right|^{2 \Delta_{\sigma}}} .
$$

The two-point functions of the operators $O_{i}(3)$

$$
\left\langle\mathcal{O}_{i} \mathcal{O}_{j}\right\rangle=\delta_{i j} \frac{N_{i}}{|x|^{2 \Delta_{i}}},
$$

where

$N_{1}=8 N^{2}\left(1+\frac{2}{N}\right), \quad N_{2}=4 N, \quad N_{3}=24$.

Similarly, the three-point functions

$$
\begin{aligned}
\left\langle\mathcal{O}_{i} \mathcal{O}_{j} \mathcal{O}_{k}\right\rangle & =\frac{C_{i j}^{k} N_{k}}{\left|x_{12}\right|^{\Delta-2 \Delta_{k}}\left|x_{23}\right|^{\Delta-2 \Delta_{i}}\left|x_{13}\right|^{\Delta-2 \Delta_{j}}}, \\
\Delta & =\Delta_{i}+\Delta_{j}+\Delta_{k}
\end{aligned}
$$

are fixed by the OPE coefficients (we list only nonzero ones)

$C_{11}^{1}=8(N+8), \quad C_{22}^{1}=2, \quad C_{12}^{2}=4(N+2)$,

$C_{22}^{2}=16, \quad C_{23}^{2}=12, \quad C_{22}^{3}=2 N, \quad C_{33}^{3}=72$.

They are related by

$$
C_{i j}^{k}=C_{i k}^{j} N_{j} / N_{k}
$$

At finite temperature two-point function takes the form

$$
\left\langle\phi_{a} \phi_{b}\right\rangle=\sum_{m=-\infty}^{\infty} \frac{\delta_{a b}}{\left[(\tau+m \beta)^{2}+\vec{x}^{2}\right]^{\Delta_{\phi}}},
$$

and similarly for $\sigma$. From here one trivially finds Eq. (8).

[1] O. Bratteli and D.W. Robinson, Operator Algebras and Quantum Statistical Mechanics: Volume 1: $C^{*}$-and $W^{*}$-Algebras. Symmetry Groups. Decomposition of States (Springer Science \& Business Media, New York \& Heidelberg, 2012).

[2] S. Weinberg, Gauge and global symmetries at high temperature, Phys. Rev. D 9, 3357 (1974).

[3] N. D. Mermin and H. Wagner, Absence of Ferromagnetism or Antiferromagnetism in One-Dimensional or TwoDimensional Isotropic Heisenberg Models, Phys. Rev. Lett. 17, 1133 (1966). 
[4] P. C. Hohenberg, Existence of long-range order in one and two dimensions, Phys. Rev. 158, 383 (1967).

[5] N. D. Mermin, Crystalline order in two dimensions, Phys. Rev. 176, 250 (1968).

[6] B. I. Halperin, On the Hohenberg-Mermin-Wagner theorem and its limitations, J. Stat. Phys. 175, 521 (2019).

[7] S. R. Coleman, There are no Goldstone bosons in twodimensions, Commun. Math. Phys. 31, 259 (1973).

[8] F. J. Dyson, Existence of a phase transition in a onedimensional Ising ferromagnet, Commun. Math. Phys. 12, 91 (1969).

[9] F. J. Dyson, Non-existence of spontaneous magnetization in a one-dimensional Ising ferromagnet, Commun. Math. Phys. 12, 212 (1969).

[10] F. J. Dyson, An Ising ferromagnet with discontinuous longrange order, Commun. Math. Phys. 21, 269 (1971).

[11] N. Laflorencie, I. Affleck, and M. Berciu, Critical phenomena and quantum phase transition in long range Heisenberg antiferromagnetic chains, J. Stat. Mech. (2005) P12001.

[12] M. F. Maghrebi, Z.-X. Gong, and A. V. Gorshkov, Continuous Symmetry Breaking in 1d Long-Range Interacting Quantum Systems, Phys. Rev. Lett. 119, 023001 (2017).

[13] N. Chai, S. Chaudhuri, C. Choi, Z. Komargodski, E. Rabinovici, and M. Smolkin, Thermal order in conformal theories, Phys. Rev. D 102, 065014 (2020).

[14] N. Chai, S. Chaudhuri, C. Choi, Z. Komargodski, E. Rabinovici, and M. Smolkin, Symmetry Breaking at All Temperatures, Phys. Rev. Lett. 125, 131603 (2020).

[15] N. Chai, E. Rabinovici, R. Sinha, and M. Smolkin, The biconical vector model at $1 / N$, J. High Energy Phys. 05 (2021) 192.

[16] S. Chaudhuri, C. Choi, and E. Rabinovici, Thermal order in large N conformal gauge theories, J. High Energy Phys. 04 (2021) 203.

[17] S. Chaudhuri and E. Rabinovici, Symmetry breaking at high temperatures in large $\mathrm{N}$ gauge theories, J. High Energy Phys. 08 (2021) 148.

[18] M. Hogervorst, S. Rychkov, and B. C. van Rees, Unitarity violation at the Wilson-Fisher fixed point in $4-\epsilon$ dimensions, Phys. Rev. D 93, 125025 (2016).

[19] B. Bajc, A. Lugo, and F. Sannino, Asymptotically free and safe fate of symmetry nonrestoration, Phys. Rev. D 103, 096014 (2021).

[20] D. F. Litim and F. Sannino, Asymptotic safety guaranteed, J. High Energy Phys. 12 (2014) 178.

[21] D. Carmi, L. Di Pietro, and S. Komatsu, A study of quantum field theories in AdS at finite coupling, J. High Energy Phys. 01 (2019) 200.

[22] A. Buchel and C. Pagnutti, Exotic hairy black holes, Nucl. Phys. B824, 85 (2010).

[23] A. Donos and J. P. Gauntlett, Superfluid black branes in $\mathrm{AdS}_{4} \times S^{7}$, J. High Energy Phys. 06 (2011) 053.

[24] U. Gürsoy, E. Kiritsis, F. Nitti, and L. S. Pimenta, Exotic holographic RG flows at finite temperature, J. High Energy Phys. 10 (2018) 173.

[25] A. Buchel, Klebanov-Strassler black hole, J. High Energy Phys. 01 (2019) 207.

[26] A. Buchel, Thermal order in holographic CFTs and no-hair theorem violation in black branes, Nucl. Phys. B967, 115425 (2021).
[27] A. Buchel, Holographic conformal order in supergravity, Phys. Lett. B 814, 136111 (2021).

[28] A. Buchel, Fate of the conformal order, Phys. Rev. D 103, 026008 (2021).

[29] A. Buchel, Compactified holographic conformal order, Nucl. Phys. B973, 115605 (2021).

[30] S.-I. Hong and J. B. Kogut, Symmetry nonrestoration in a Gross-Neveu model with random chemical potential, Phys. Rev. D 63, 085014 (2001).

[31] Z. Komargodski, A. Sharon, R. Thorngren, and X. Zhou, Comments on Abelian Higgs models and persistent order, SciPost Phys. 6, 003 (2019).

[32] K. Aitken, A. Cherman, E. Poppitz, and L. G. Yaffe, QCD on a small circle, Phys. Rev. D 96, 096022 (2017).

[33] Y. Tanizaki, T. Misumi, and N. Sakai, Circle compactification and 't Hooft anomaly, J. High Energy Phys. 12 (2017) 056.

[34] G. V. Dunne, Y. Tanizaki, and M. Ünsal, Quantum distillation of Hilbert spaces, semi-classics and anomaly matching, J. High Energy Phys. 08 (2018) 068.

[35] Z. Wan and J. Wang, Higher anomalies, higher symmetries, and cobordisms III: QCD matter phases anew, Nucl. Phys. B957, 115016 (2020).

[36] V. L. Berezinsky, Destruction of long range order in onedimensional and two-dimensional systems having a continuous symmetry group. I. Classical systems, Sov. Phys. JETP 32, 493 (1971), https://s3.cern.ch/inspire-prod-files-f/ f55503250f690969aedfda4ceaf9b4f9.

[37] V. L. Berezinsky, Destruction of long-range order in Onedimensional and two-dimensional systems possessing a continuous symmetry group. II. Quantum systems. Sov. Phys. JETP 34, 610 (1972), https://inspirehep.net/files/ Of7b50c47ec26bed99a50ff199960259.

[38] J. M. Kosterlitz and D. J. Thouless, Ordering, metastability and phase transitions in two-dimensional systems, J. Phys. C 6, 1181 (1973).

[39] M. F. Paulos, S. Rychkov, B. C. van Rees, and B. Zan, Conformal invariance in the long-range Ising model, Nucl. Phys. B902, 246 (2016).

[40] N. Chai, A. Dymarsky, M. Goykhman, R. Sinha, and M. Smolkin, A model of persistent breaking of continuous symmetry, arXiv:2111.02474.

[41] M. E. Fisher, S.-k. Ma, and B. G. Nickel, Critical Exponents for Long-Range Interactions, Phys. Rev. Lett. 29, 917 (1972).

[42] J. Sak, Recursion relations and fixed points for ferromagnets with long-range interactions, Phys. Rev. B 8, 281 (1973).

[43] J. Honkonen and M. Y. Nalimov, Crossover between field theories with short-range and long-range exchange or correlations, J. Phys. A 22, 751 (1989).

[44] Accurate critical exponents for ising like systems in noninteger dimensions, In Large-Order Behaviour of Perturbation Theory, edited by J. C. Le Guillou and J. Zinn-Justin, Current Physics-Sources and Comments Vol. 7 (Elsevier, New York, 1990), pp. 559-564.

[45] S. El-Showk, M. Paulos, D. Poland, S. Rychkov, D. Simmons-Duffin, and A. Vichi, Conformal Field Theories in Fractional Dimensions, Phys. Rev. Lett. 112, 141601 (2014).

[46] E. Luijten and H. W. J. Blöte, Boundary between LongRange and Short-Range Critical Behavior in Systems with Algebraic Interactions, Phys. Rev. Lett. 89, 025703 (2002). 
[47] M. Picco, Critical behavior of the Ising model with long range interactions, arXiv:1207.1018.

[48] T. Blanchard, M. Picco, and M. A. Rajabpour, Influence of long-range interactions on the critical behavior of the Ising model, Europhys. Lett. 101, 56003 (2013).

[49] M. C. Angelini, G. Parisi, and F. Ricci-Tersenghi, Relations between short-range and long-range ising models, Phys. Rev. E 89, 062120 (2014).
[50] N. Defenu, A. Trombettoni, and A. Codello, Fixed-point structure and effective fractional dimensionality for $\mathrm{o}(\mathrm{n})$ models with long-range interactions, Phys. Rev. E 92, 052113 (2015).

[51] C. Behan, L. Rastelli, S. Rychkov, and B. Zan, Long-Range Critical Exponents Near the Short-Range Crossover, Phys. Rev. Lett. 118, 241601 (2017). 